NARRATIVE REVIEW

Volume 2 (2021), No. 2, pp.67-77

DOI: $10.15367 /$ ch.v2i2.486

\title{
Child Sexual Abuse Recurrence: A Narrative Review
}

\author{
RACHEL WILDFEUER ${ }^{1}$; JULIA M. KOBULSKY, PHD²; JOSÉ N. REYES III ${ }^{3}$ \\ ${ }^{1}$ Department of Sociology, Temple University \\ ${ }^{2}$ School of Social Work, College of Public Health, Temple University \\ ${ }^{3}$ Department of Health Services Administration and Policy, College of Public Health, Temple University \\ Correspondence: rachel.wildfeuer@,temple.edu (Rachel Wildfeuer)
}

\begin{abstract}
Child sexual abuse recurrence can have a detrimental and devastating effect on victims. Less is known, however, about the risk and protective factors associated with child sexual abuse recurrence. In order to better understand these risk and protective factors, a narrative review was conducted of the current literature. Academic databases, cited references, and reference lists were searched for peer-reviewed research that focused on sexual abuse recurrence prior to age 18 years. The risk and protective factors identified in the literature were organized into four a priori categories based on an ecological systems framework: child, characteristics of the maltreatment, family, and community/social systems. Examples of risk factors at each level were child mental health concerns (child), the perpetrator being the mother's significant other (characteristics of the maltreatment), parental history of sexual abuse (family), and active or prior child welfare involvement (community/social systems). Only two protective factors were identified, both regarding the family: family social support and a parent believing the child's report of the initial victimization. There were mixed or inconclusive findings for several factors. Overall, the review indicates that tertiary prevention, including child-, caregiver-, and perpetrator-focused strategies, should be employed to mitigate the risk factors and enhance the protective factors for child sexual abuse recurrence. Further research is needed to address protective factors and community/social systems factors and could, building on this narrative review, involve a systematic review of the child sexual abuse recurrence literature.
\end{abstract}

\section{Introduction}

Child sexual abuse is a significant, costly social problem that threatens wellbeing in multiple dimensions throughout the lifespan. ${ }^{1-7}$ Rates of child sexual abuse have declined, ${ }^{8}$ but it remains common with a lifetime prevalence of $4 \%$ for Child Protective Services (CPS)-investigated child sexual abuse in the United States. ${ }^{9}$ Child sexual abuse is associated with a range of physical and mental health problems including post-traumatic stress, mood, anxiety, and substance use disorders ${ }^{10,11}$; sexual risk behaviors, obesity and pain; and gastrointestinal, gynecologic and cardio-pulmonary symptoms. ${ }^{1-6,12}$ Child sexual abuse is additionally associated with subsequent risks for violence perpetration and victimization. ${ }^{13}$ The lifetime costs of child sexual abuse occurring in the United States in 2015, including those related to health care, child welfare and criminal justice systems, special education, death, and productivity losses were estimated at $\$ 9.3$ million. $^{7}$ 
The consequences of child sexual abuse necessitate its prevention. A meta-analytic review of risk and protective factors for child sexual abuse found that prior sexual abuse exposure is its strongest predictor. ${ }^{14}$ While the rate of child sexual abuse recurrence in research varies depending on sample characteristics and how recurrence is measured, findings suggest that $17-39 \%$ of child sexual abuse victims experience recurrence during childhood or adolescence. ${ }^{15,16}$ Among other problems, studies have found that recurrence is associated with later difficulties in interpersonal relationships and acute psychiatric problems. ${ }^{17,18}$ Thus, child sexual abuse prevention initiatives should include tertiary prevention focused on eliminating revictimization among children who have already experienced sexual abuse.

Effective tertiary prevention should be guided by empirical knowledge of the risk and protective factors for child sexual abuse recurrence. A past narrative review ${ }^{17}$ and a meta-analysis ${ }^{19}$ of child sexual abuse recurrence have combined studies on recurrence of sexual victimization in childhood and adulthood. Therefore, previous reviews do not provide a clear picture of childhood recurrence of sexual abuse. Moreover, the past narrative review examining risk and protective factors for recurrence is not current, including literature only through 2015.17 The narrative review suggested that individual factors (e.g., psychosocial and behavioral functioning), family (e.g., parental substance use), perpetrators (e.g., a male perpetrator), and engagement with helping professionals (e.g., therapeutic support) may mediate risk of recurrence. ${ }^{17}$ The meta-analysis examined the roles of child age and gender, finding no support that either affects recurrence. ${ }^{19}$ In order to inform tertiary prevention, this narrative review was conducted of the current literature to gather information on risk and protective factors for child sexual abuse recurrence.

\section{Methods}

In the fall of 2020, academic literature was searched using keywords such as "child sexual abuse", "child sexual assault", "recurrence", "revictimization", "recidivism", and "chronicity" within Web of Science and Google Scholar, using cited reference searches, and using sources from reference lists. The search concentrated on research on sexual abuse recurrence until age 18. Literature was reviewed and summarized using an ecological systems framework to identify common themes. ${ }^{20,21}$ An ecological systems framework suggests that factors in the child's immediate environment and more distal contextual factors contribute to recurrence. ${ }^{20,21}$ The factors associated with the risk of recurrence were organized into four a priori categories: child-level characteristics, characteristics of the maltreatment, family-level characteristics, and characteristics of the community/social systems.

\section{Results}

Table 1 summarizes the main findings regarding the factors associated with child sexual abuse recurrence. The literature suggests certain characteristics of the child, maltreatment, family, and community/social systems are associated with child sexual abuse recurrence risk and that certain familial characteristics are protective. Additionally, findings were mixed or inconclusive regarding select characteristics of the child, family, and community/social systems. 


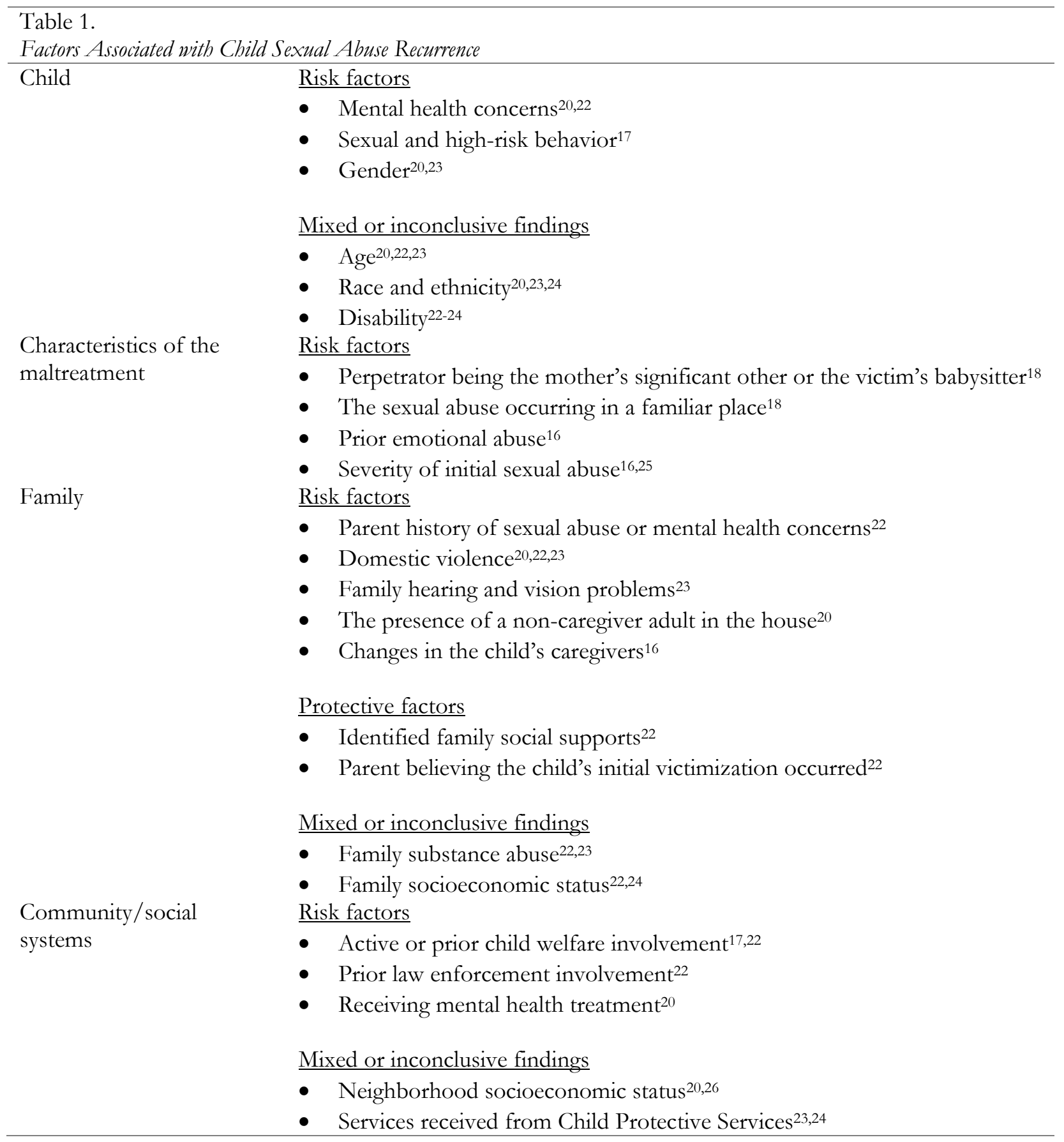

\section{Child}

Research found that child mental health concerns, sexual and high-risk behavior, and gender are related to risk of recurrence. Mental health concerns, defined as both a report of any mental health problem (i.e., single item from case records $)^{20}$ and as a mental health diagnosis (i.e., depression, anxiety, attention deficit disorder, bipolar disorder, oppositional defiant disorder, mood disorder), ${ }^{22}$ were associated with higher risk of recurrence, as were sexual and high-risk behaviors, such as having sex with anonymous partners 
and increased alcohol and substance use. ${ }^{17}$ Mental health concerns caused by initial victimization and resultant coping mechanisms may increase children's vulnerability for recurrence. ${ }^{17}$ Additionally, girls have been shown to be at higher risk for sexual abuse recurrence. ${ }^{20,23}$ These gender differences may be due, however, to the fact that sexual abuse recurrence studies generally include larger samples of girls than boys and that sexual abuse is generally more prevalent among girls. ${ }^{20}$

Research shows mixed or inconclusive findings regarding child age, race and ethnicity, and disability. Two studies, both of which retrospectively reviewed records from children ages 0-18 years presenting to children's advocacy centers for assessments, suggest children victimized at a younger age are most at risk for recurrence. ${ }^{20,22}$ One of these studies restricted recurrence to situations when victimization was by a different perpetrator ${ }^{20}$ and the other examined bivariate relationships. ${ }^{22}$ Another study, which employed a prospective longitudinal design with data based on substantiated CPS reports from 45 states, found that younger child age was associated with decreased risk for recurrence. ${ }^{23}$ This study had a larger and more geographically dispersed sample size than the two studies that found associations between younger age and increased risk for recurrence.

Similarly, research regarding the role of race or ethnicity had mixed findings. One study, which retrospectively reviewed records from a children's advocacy center, suggested that racial-ethnic minority children (African American, Native American, Hispanic, Asian American, or Middle Eastern) had a higher risk for subsequent sexual abuse than White non-Hispanic children. ${ }^{20}$ Of note, this study had a sample that was $79 \%$ White non-Hispanic and used a binary measure that conflated race and ethnicity. ${ }^{20}$ Two studies analyzing data from substantiated CPS reports and using relatively granular measures of race/ethnicity, indicated that Hispanic ethnicity is a protective factor against recurrence but found no racial differences. ${ }^{23,24}$ These studies had samples that were $64.7 \%$ White and from 45 states $^{23}$ and $62 \%$ White and from seven states, ${ }^{24}$ respectively.

Research regarding child disability has also been inconclusive. A retrospective chart review at a children's advocacy center found a bivariate relationship between presence of a diagnosed developmental delay/intellectual disability and recurrence. ${ }^{22}$ Another study, which employed a prospective longitudinal design with data based on substantiated CPS reports from 45 states, found bivariate relationships between presence of learning problems, medical problems, and any disability (intellectual, learning, physical, or other disability, visual impairment, or medical problems) and sexual abuse recurrence. However, these relationships were not significant in multivariate models analyzing other child, family, offender, and report characteristics. Thus, the associations between learning problems, medical problems, and any disability and sexual abuse recurrence appear to be attenuated by these other characteristics. ${ }^{23}$ On the other hand, child disability (intellectual disability, emotional disturbance, visual impairment, physical disability, or behavioral problems) was associated with decreased likelihood for reports of recurrence when utilizing prospective data of substantiated CPS reports from seven states. ${ }^{24}$ This study excluded children who were placed into out-of-home care, which may explain conflicting findings.

In summary, prior research indicates that child mental health concerns, defined both as a report of any mental health problem and as a mental health diagnosis, are associated with higher risk of recurrence. Sexual and high-risk behavior, including having sex with anonymous partners and increased alcohol and substance use, are also related to higher risk of recurrence. Finally, girls have been shown to be at higher risk for recurrence. Research is mixed or inconclusive regarding child age, race and ethnicity, and disability. Of note, studies with stronger methods (i.e., prospective designs, comprehensive measures of recurrence, large/diverse samples) found that younger age and Hispanic ethnicity were associated with lower sexual abuse recurrence and they found no disability effects on recurrence. 


\section{Characteristics of the Maltreatment}

Research indicates that characteristics of the maltreatment, including the relationship of the perpetrator to the victim, the geographic location of the sexual abuse, prior emotional abuse, and maltreatment severity are related to risk of recurrence. There is a higher risk of recurrence for perpetrators who are the victim's stepfather, mother's boyfriend, or babysitter, compared to perpetrators who are strangers. ${ }^{18}$ Sexual abuse that occurs in a familiar place, defined as the victim's home, the babysitter's home, or the victim's school, relative to an unfamiliar place, is also associated with risk of recurrence. ${ }^{18}$ Additionally, prior emotional abuse was related to risk of recurrence in a prospective study that analyzed substantiated child sexual abuse reports from two children's hospital's Child Protection Units in Sydney, Australia. ${ }^{16}$ This association could be due to the fact that reports of emotional abuse often reflect concerns about poor family functioning. ${ }^{16}$

The severity of initial sexual abuse is also associated with recurrence. Two prospective studies, one that measured recurrence with substantiated reports in Australia and another that used retrospective selfreports in Christchurch, New Zealand, suggest that more severe childhood sexual abuse is associated with increased risk of recurrence. ${ }^{16,25}$ The Australian study measured maltreatment severity with four categories of increasing severity: attempted indecent assault (i.e., inappropriate touching), indecent assault, attempted sexual assault, and sexual assault. ${ }^{16}$ The New Zealand study also measured maltreatment severity with four categories: no childhood sexual abuse, noncontact childhood sexual abuse (e.g., indecent exposure), contact sexual abuse involving inappropriate touching, and contact sexual abuse involving attempted or completed sexual intercourse. ${ }^{25}$

In summary, research suggests that there is a higher risk of recurrence associated with perpetrators who are the mother's significant other or the victim's babysitter. Sexual abuse that takes place in a location familiar to the child is also related to risk of recurrence. Finally, prior emotional abuse and more severe childhood sexual abuse is associated with higher risk of recurrence.

\section{Family}

Research suggests that certain aspects of the family are related to risk of recurrence, these include parental characteristics and household characteristics. Parental history of sexual abuse and parental mental health concerns are family risk factors for child sexual abuse recurrence. ${ }^{22}$ Domestic violence $20,22,23$ and family hearing and vision problems ${ }^{23}$ are also associated with risk of recurrence. Additionally, the presence of a non-caregiver adult in the house ${ }^{20}$ and changes in the child's caregivers ${ }^{16}$ are family risk factors for sexual abuse recurrence. Family violence and dysfunction are potential barriers to children disclosing sexual abuse and seeking help after victimization, which may put them at increased risk for recurrence. ${ }^{20}$

In terms of family protective factors, research that retrospectively analyzed records from a children's advocacy center found bivariate relationships between the family having an identified support system, the child having a supportive parent who believes the first victimization occurred,, and lower risk of sexual abuse recurrence. ${ }^{22}$ Research suggests that caregiver support promotes healthy adjustment following child sexual abuse victimization and may influence a child's decision to disclose sexual abuse. ${ }^{17}$

The literature is mixed or inconclusive regarding family substance abuse and family socioeconomic status (SES). A study employing a retrospective design with records from a children's advocacy center suggests a bivariate association between parental drug and alcohol concerns and higher risk of recurrence. ${ }^{22}$ However, a prospective study using data from substantiated CPS reports in 45 states found that family substance abuse was associated with decreased risk of recurrence in multivariate models analyzing child, 
family, offender, and report characteristics. ${ }^{23}$ Similarly, retrospective analysis of records from a children's advocacy center found bivariate relationships between family financial concerns, receiving public assistance, and higher risk of recurrence. ${ }^{22}$ On the other hand, an analysis of prospective data on substantiated CPS reports among children remaining in their homes from seven states found that family financial problems (i.e., inability to provide sufficient financial resources to meet minimum basic needs) were associated with decreased likelihood for reports of recurrence. ${ }^{24}$

In summary, research indicates that parental history of sexual abuse, parental mental health concerns, domestic violence, and family hearing and vision problems are associated with higher risk of sexual abuse recurrence. The presence of a non-caregiver in the house and changes in the child's caregivers are also related to risk of recurrence. Identified family social supports and parents who accept children's initial reports of victimization are protective factors against recurrence. Research is mixed regarding family substance use, with a methodologically stronger study suggesting its association with lower recurrence. Previous research is inconclusive regarding family SES and recurrence of sexual abuse in childhood.

\section{Community/Social Systems}

The reviewed studies indicated that certain community/social systems factors, including child welfare involvement, law enforcement involvement, and mental health treatment are related to risk of recurrence. Active or prior child welfare involvement, particularly foster care placement, and families' prior law enforcement involvement have been associated with higher sexual abuse recurrence. ${ }^{17,22}$ Parents who are unable to comply with child welfare mandates or recommendations may place their children at risk for recurrence by prolonging their involvement in the child welfare system. ${ }^{17}$ Additionally, families with child welfare involvement and/or law enforcement involvement may also struggle with higher levels of violence and other co-occurring risk factors. Receiving psychotherapy is also positively associated with sexual abuse recurrence for children. This is likely attributable to the fact that children with mental health concerns are more likely to be in psychotherapy and receive mental health treatment. ${ }^{20}$

There are mixed or inconclusive findings regarding both neighborhood SES and services received from CPS. Youth living in census tracts with lower median incomes had higher rates of recurrence than those living in higher income neighborhoods, when prospective data based on reports from the Missouri Division of Family Services were analyzed. ${ }^{26}$ However, another study found, that neighborhood median household income and educational attainment were not related to recurrence when retrospectively analyzing records from a children's advocacy center. ${ }^{20}$ In this study, neighborhoods were measured at the zip code level, which may have had limited variability in the local sample, and only bivariate relationships were examined. ${ }^{20}$

In terms of services received from CPS, a prospective analysis of data based on substantiated CPS reports from seven states found that receiving family supportive services (i.e., community-based preventative activities) was associated with sexual abuse recurrence compared to not receiving family supportive services. On the other hand, receiving family preservation services (i.e., activities designed to help families alleviate crises) from CPS, compared to not receiving family preservation services, was associated with lower risk of recurrence as was receiving both family supportive services and family preservation services from CPS compared to not receiving both services. ${ }^{24}$ These differences may be due to variation amongst families who were assigned to receive different services. ${ }^{24}$ Of note, another study which prospectively analyzed data based on substantiated CPS reports from 45 states found that none of the referrals reduced sexual abuse recurrence although there was a trend toward risk reduction after receiving a referral for substance abuse services. ${ }^{23}$ 
In summary, research suggests that active or prior child welfare involvement and prior law enforcement involvement are associated with higher risk of recurrence. Receiving mental health treatment is also related to risk of recurrence. Although there were mixed findings neighborhood SES, low SES measured at the census tract-level versus the zip code-level predicted child sexual abuse recurrence. Research is inconclusive regarding the relationship between services received from CPS and recurrence.

\section{Discussion}

\section{Summary}

The findings of this narrative review revealed certain characteristics of the child, maltreatment, family, and community/social systems that are associated with child sexual abuse recurrence. Risk factors related to the child are mental health concerns, sexual and high-risk behavior, and being a girl. Risk factors related to characteristics of the maltreatment are a perpetrator who is the victim's babysitter or the significant other of the victim's mother, sexual abuse that occurs in a familiar place, prior emotional abuse, and severity of initial sexual abuse. Risk factors related to the family are parental history of sexual abuse or mental health concerns, domestic violence, family hearing and vision problems, the presence of a noncaregiver adult in the home, and changes in the child's caregivers. Lastly, risk factors related to the community/social systems are active or prior child welfare involvement, prior law enforcement involvement, and receiving mental health treatment.

Protective factors identified in the literature were few, and only occurred in the context of the family. The protective factors were clearly identified family social supports and the parent believing the authenticity of the child's initial sexual abuse. There were mixed or inconclusive findings regarding child age, child race and ethnicity, child disability, family substance abuse, family SES, neighborhood SES, and services received from CPS. Mixed and inconclusive findings potentially occurred due to methodological differences among studies such as overall design, variable operationalization, and sampling.

\section{Practical Implications}

Findings highlight distinct risk factors for child sexual abuse recurrence that may be mitigated by tertiary prevention, as well as protective factors that may be enhanced. Tertiary prevention aims to prevent the recurrence of maltreatment by reducing the harms associated with initial victimization. ${ }^{27}$ Child-, caregiver, and perpetrator-focused strategies are needed to effectively prevent child sexual abuse and its recurrence. Research suggests that trauma-focused psychotherapies, including trauma-focused cognitive behavioral therapy, help improve the negative psychological effects and mental health symptoms associated with childhood sexual abuse. ${ }^{28-30}$ Given evidence suggesting that child mental health symptoms increase risk for recurrence, ${ }^{17,20,22}$ such strategies may serve as tertiary prevention.

Additionally, given the connections between family dysfunction, family instability, and recurrence, ${ }^{20,22,23}$ tertiary prevention should address the impact of the family environment. Engaging caregivers in a child sexual abuse prevention program can enhance their awareness of childhood sexual abuse and their preparation to utilize protective behavioral strategies such as monitoring one-on-one time between the child and adults and vetting babysitters. ${ }^{31}$

Comprehensive or "wraparound" approaches deserve research attention as potential strategies to reduce recurrence by addressing multiple, interrelated risk factors identified in our narrative review (e.g., parental 
and child mental health problems and trauma) with an individualized, family-centered approach. ${ }^{32}$ For example, the Child Protective Services Reintegration Project (CPSR) is an evidence-based, familycentered wraparound program that works to promote permanency in the home for children with severe mental health issues. ${ }^{33}$ Program staff work with families to set goals that are specific to their perceived emotional, physical (including concrete and financial supports), and parenting needs, as well as their child's needs. The program staff also connect families with any applicable services including social support programs, local medical and mental health services, and ongoing case management options. This program does not specifically focus on children who are victims of sexual abuse. However, the highly personalized nature of the program and goal-setting process allows it to adapt to each child and family's needs. Wraparound services like CPSR, which focus on child mental health, multifaceted family support, and parenting training, may reduce multiple risk factors for child sexual abuse recurrence identified in this review.

Recent research argues that a focus on perpetrators, through targeted interventions and specialized trainings for clinical therapists, is also needed for a more comprehensive approach to preventing child sexual abuse and its recurrence..$^{34,35}$ This is important, especially given evidence that a quarter of recurrent sexual abuse cases involve the same offender. ${ }^{23}$ The few existing programs focused on perpetrators focus on individual or small group programming with highly tailored goals. ${ }^{34,36}$ In general, there is a need for greater focus on the development and wider dissemination of these interventions.

The Problematic Sexual Behavior - Cognitive-Behavioral Treatment (PSB-CBT) program seeks to reduce further perpetration of child sexual abuse by prior youth offenders. ${ }^{36}$ Programs like PSB-CBT address specific risk and protective factors identified in this narrative review including child sexual and high-risk behaviors and family social supports. The program provides group treatment for youth and their caregivers in both concurrent and combined multi-family sessions. PSB-CBT provides youth with education regarding rules about sexual behavior and skills including coping, self-control, and decision making. Caregivers are instructed in managing child behavior, supporting healthy development, sex education, abuse prevention, and rules about sexual behavior. Program evaluation has indicated significant and rapid reductions in problematic sexual behavior among youth participants, increased parenting skills, and decreased parenting stress. Benefits for the program were equivalent among participants regardless of ethnicity and geographic location. Lastly, these gains were sustained post treatment. ${ }^{36}$

\section{Research Implications and Conclusions}

In light of these findings, further research should address protective factors and community/social systems factors. The narrative review only identified two protective factors and inconsistent findings regarding community/social systems factors. In line with an ecological systems framework, the public health approach to child sexual abuse prevention emphasizes the role of community and neighborhood factors along with individual, family, and system factors. ${ }^{37}$ Additionally, findings indicate that the role of child welfare system services in preventing recurrence is not well understood. These relationships should be clarified in research that addresses potentially important differences in the levels of risk among youth who are referred to different types of services. Methodologically, the studies in the narrative review overwhelmingly rely on administrative data, which are particularly prone to surveillance bias and nondetection. ${ }^{38}$ Future studies should integrate multiple sources (i.e., self-report and administrative data) to reduce in error in child sexual abuse recurrence measurement. Further, many of the studies were based on retrospective chart reviews ${ }^{18,20,22}$ versus prospective, longitudinal designs which can clarify temporal sequence. ${ }^{16,23,24}$ Finally, the ecological systems framework utilized in this narrative review and its consideration of both risk and protective factors can provide a foundation for a broadened and systematic 
literature review. A systematic review could allow for the identification of additional studies potentially missed in our search strategy, providing additional clarification on patterns in the literature and discrepancies.

This narrative review provides an assessment of current knowledge regarding child sexual abuse recurrence. Moreover, this review distilled risk factors for sexual abuse recurrence at multiple levels of the social ecology, and family-level protective factors. These findings can provide a blueprint for both researchers and child welfare practitioners seeking to advance prevention that will reduce the recurrence of child sexual abuse.

\section{Disclosures and Conflicts of Interest}

The authors have no conflicts to disclose.

\section{Acknowledgments}

We thank the Philadelphia Children's Alliance, Bernie Newman, Devon Della-Croce, and Cameron O'Donnell for their collaboration in developing this narrative review.

\section{References}

1. Meyer D, Cohn A, Robinson B, Muse F, Hughes R. Persistent complications of child sexual abuse: Sexually compulsive behaviors, attachment, and emotions. J Child Sex Abus. 2017;26(2):140-157. doi:10.1080/10538712.2016.1269144

2. Fisher HL, Jones PB, Fearon P, et al. The varying impact of type, timing and frequency of exposure to childhood adversity on its association with adult psychotic disorder. Psychol Med. 2010;40(12):1967-1978. doi:10.1017/S0033291710000231

3. Brunton R, Dryer R. Child sexual abuse and pregnancy: A systematic review of the literature. Child Abuse Negl. 2020;111: 10482. doi:10.1016/j.chiabu.2020.104802

4. Easton SD, Kong J. Mental health indicators fifty years later: A population-based study of men with histories of child sexual abuse. Child Abuse Negl. Jan 2017;63:273-283. doi:10.1016/j.chiabu.2016.09.011

5. Papalia NL, Luebbers S, Ogloff JRP, Cutajar M, Mullen PE. The long-term co-occurrence of psychiatric illness and behavioral problems following child sexual abuse. Aust N Z J Psychiatry. Jun 2017;51(6):604-613. doi:10.1177/0004867416667232

6. Sanjeevi J, Houlihan D, Bergstrom KA, Langley MM, Judkins J. A review of child sexual abuse: Impact, risk, and resilience in the context of culture. J Child Sex Abus. 2018;27(6):622-641. doi:10.1080/10538712.2018.1486934

7. Letourneau EJ, Brown DS, Fang XM, Hassan A, Mercy JA. The economic burden of child sexual abuse in the United States. Child Abuse Negl. May 2018;79:413-422. doi:10.1016/j.chiabu.2018.02.020

8. Finkelhor D, Saito K, Jones L. Updated trends in child maltreatment, 2018. Crimes Against Children Research $\quad 2020 . \quad$ http://unh.edu/ccrc/pdf/CV203\%20\%20Updated $\% 20$ trends $\% 202018 \_k s \_d f . p d f$

9. Kim H, Wildeman C, Jonson-Reid M, Drake B. Lifetime prevalence of investigating child maltreatment among US children. Am J Public Health. Feb 2017;107(2):274-280. doi:10.2105/ajph.2016.303545 
10. Noll JG. Child sexual abuse as a unique risk factor for the development of psychopathology: The compounded convergence of mechanisms. Annu Rev Clin Psychol. 2021;17:439-464. doi:10.1146/annurev-clinpsy-081219-112621

11. Irish L, Kobayashi I, Delahanty DL. Long-term physical health consequences of childhood sexual abuse: A meta-analytic review. J Pediatric Psychol. Jun 2010;35(5):450-461. doi:10.1093/jpepsy/jsp118

12. Schroeder K, Schuler BR, Kobulsky JM, Sarwer DB. The association between adverse childhood experiences and childhood obesity: A systematic review. Obes Rev. Jul 2021;22(7):e13204. doi:10.1111/obr.13204

13. Voith LA, Anderson RE, Cahill SP. Extending the ACEs framework: Examining the relations between childhood abuse and later victimization and perpetration with college men. J Interpers Violence. Sep 2020;35(17-18):3487-3512. doi:10.1177/0886260517708406

14. Assink M, van der Put CE, Meeuwsen M, et al. Risk factors for child sexual abuse victimization: A meta-analytic review. Psychol Bull. May 2019;145(5):459-489. doi:10.1037/bul0000188

15. Finkelhor D, Ormrod RK, Turner HA. Re-victimization patterns in a national longitudinal sample of children and youth. Child Abuse Negl. May 2007;31(5):479-502. doi:10.1016/j.chiabu.2006.03.012

16. Swanston HY, Parkinson PN, Oates RK, O’Toole BI, Plunkett AM, Shrimpton S. Further abuse of sexually abused children. Child Abuse Negl. 2002;26(2):115-127. doi: 10.1016/s0145-2134(01)003118

17. Pittenger SL, Huit TZ, Hansen DJ. Applying ecological systems theory to sexual revictimization of youth: A review with implications for research and practice. Aggress Violent Behav. Jan-Feb 2016;26:35-45. doi:10.1016/j.avb.2015.11.005

18. Hu MH, Huang GS, Huang JL, et al. Clinical characteristic and risk factors of recurrent sexual abuse and delayed reported sexual abuse in childhood. Medicine. Apr 2018;97(14):e0236. doi: $10.1097 / \mathrm{md} .0000000000010236$

19. Walker HE, Freud JS, Ellis RA, Fraine SM, Wilson LC. The prevalence of sexual revictimization: A meta-analytic review. Trauma Violence Abuse. Jan 2019;20(1):67-80. doi:10.1177/1524838017692364

20. Pittenger SL, Pogue JK, Hansen DJ. Predicting sexual revictimization in childhood and adolescence: A longitudinal examination using ecological systems theory. Child Maltreat. 2018;23(2):137-146. doi: 10.1177/1077559517733813

21. Belsky J. Child maltreatment: An ecological integration. Am Psychol. 1980;35(4):320-335. doi:10.1037/0003-066x.35.4.320

22. Hornor G, Fischer BA. Child sexual abuse revictimization: Child demographics, familial psychosocial factors, and sexual abuse case characteristics. J Forensic Nurs. Oct/Dec 2016;12(4):151-159. doi:10.1097/jfn.0000000000000124

23. Palusci VJ, Ilardi M. Risk factors and services to reduce child sexual abuse recurrence. Child Maltreat. Feb 2020;25(1):106-116. doi:10.1177/1077559519848489

24. Sinanan AN. The impact of child, family, and child protective services factors on reports of child sexual abuse recurrence. J Child Sex Abus. 2011;20(6):657-676. doi: 10.1080/10538712.2011.622354

25. Fergusson DM, Horwood LJ, Lynskey MT. Childhood sexual abuse, adolescent sexual behaviors and sexual revictimization. Child Abuse Negl. Aug 1997;21(8):789-803. doi:10.1016/s01452134(97)00039-2

26. Drake B, Jonson-Reid M, Way I, Chung S. Substantiation and recidivism. Child Maltreat. 2003;8(4):248-260. doi:10.1177/1077559503258930

27. Jones Harden B, Simons C, Johnson-Motoyama M, Barth R. The child maltreatment prevention landscape: Where are we now, and where should we go? An Am Acad Pol Soc Sci. 2020;692(1):97118. doi:10.1177/0002716220978361 
28. Kim S, Noh D, Kim H. A summary of selective experimental research on psychosocial interventions for sexually abused children. J Child Sex Abus. Jul 2016;25(5):597-617. doi:10.1080/10538712.2016.1181692

29. Allen B, Hoskowitz NA. Structured trauma-focused CBT and unstructured play/experiential techniques in the treatment of sexually abused children: A field study with practicing clinicians. Child Maltreat. May 2017;22(2):112-120. doi:10.1177/1077559516681866

30. McTavish JR, Santesso N, Amin A, et al. Psychosocial interventions for responding to child sexual abuse: A systematic review. Child Abuse Negl. 2021/06/01/ 2021;116:104203. doi: 10.1016/j.chiabu.2019.104203

31. Guastaferro K, Felt JM, Font SA, et al. Parent-focused sexual abuse prevention: Results from a cluster randomized trial. Child Maltreat. Oct 7 2020:1077559520963870. doi:10.1177/1077559520963870

32. Reunification NQICoF-C. Family-centered reunification in child welfare: A review of best practices. 2021. https:/ / qicfamilyreunification.org/wp-content/uploads/2021/03/QICRcatalog.pdf

33. Maher, EJ, Ward, KJ, Peveto, L, McRoy, R, Cuellar, A, Madden, E. Permanency outcomes for youth with complex mental health needs served by the Child Protective Services Reintegration Project. 2011. https:// caseyfamilypro-wpengine.netdna-ssl.com/media/TravisCounty_PhaseII_ES.pdf

34. McKibbin G, Humphreys C, Hamilton B. "Talking about child sexual abuse would have helped me": Young people who sexually abused reflect on preventing harmful sexual behavior. Child Abuse Negl. Aug 2017;70:210-221. doi:10.1016/j.chiabu.2017.06.017

35. Assini-Meytin LC, Fix RL, Letourneau EJ. Child sexual abuse: The need for a perpetration prevention focus. J Child Sex Abus. Jan 2020;29(1):22-40. doi:10.1080/10538712.2019.1703232

36. Silovsky JF, Hunter MD, Taylor EK. Impact of early intervention for youth with problematic sexual behaviors and their caregivers. J Sex Aggres. Jan 2019;25(1):4-15. doi:10.1080/13552600.2018.1507487

37. McKillop N. Chapter 13 - Understanding the Nature and Dimensions of Child Sexual Abuse to Inform Its Prevention. In: Bryce I, Robinson Y, Petherick W, eds. Child Abuse and Neglect: Forensic Issues in Evidence, Impact, and Management. Academic Press; 2019:241-259. doi: 10.1016/B978-012-815344-4.00013-1

38. Kobulsky JM, Kepple NJ, Jedwab M. Abuse characteristics and the concordance of Child Protective Service determinations and adolescent self-reports of abuse. Child Maltreat. Aug 2018;23(3):269-280. doi:10.1177/1077559518771743

\section{Statement of Contributions}

Wildfeuer, Kobulsky, and Reyes wrote the paper and Wildfeuer had primary responsibility for final content. 